\title{
Lineare zirkumskripte Sklerodermie
}

\section{Linear Scleroderma}

Autoren

Institut
N. Müller, M. Konrad, C. Löser, E. Dippel

Hautklinik, Klinikum der Stadt Ludwigshafen GmbH (Direktor: Prof. Dr. E. Dippel)

\section{Bibliografie}

DOI http://dx.doi.org/

10.1055/s-0029-1243998

Online-Publikation: 16. 3. 2010

Akt Dermatol 2010; 36:

177-179 @ Georg Thieme

Verlag KG Stuttgart · New York ISSN 0340-2541

\section{Korrespondenzadresse}

\section{Dr. Nina Müller}

Hautklinik

Klinikum der Stadt Ludwigshafen $\mathrm{GmbH}$ Bremserstraße 79 67063 Ludwigshafen muellern@klilu.de

\section{Zusammenfassung \\ $\nabla$}

Die zirkumskripte lineare Sklerodermie ist eine Verlaufsform der Morphea, die durch eine lineare, meist einseitige Anordnung der Effloreszenzen an Armen und Beinen gekennzeichnet ist. Nicht selten treten bei tiefreichenden Verlaufsformen durch Beteiligung von Knochen und Muskulatur Kontrakturen auf, mit z.T. erheblichen körperlichen und auch psychischen Konsequenzen. Die Ätiologie der Erkrankung ist bisher ungeklärt. Meist ist eine Lokaltherapie mit z.B. Glukosteroiden, Vitamin-D3-Analoga, UVA1- oder PUVA-

\section{Anamnese}

Wir berichten über eine 1999 geborene, damals 9-jährige Patientin, bei der erstmalig im Juni 2007 über dem linken Ellenbogengelenk ein weißer Fleck aufgefallen war. Bei Berührung bestand vermehrte Schmerzempfindlichkeit, zudem trat in diesem Areal intermittierend ein Hitzegefühl auf. Im weiteren Verlauf zeigte sich eine kontinuierliche Verhärtung der Läsion und Progredienz mit Ausbildung weiterer Herde entlang des Unterarms und konsekutiver Beugekontraktur des linken Ringfingers im Mittelgelenk. Schwerwiegende Vorerkrankungen waren bei der jungen Patientin nicht bekannt, seit 2006/2007 bestand gelegentlich Migränesymptomatik mit halbseitigen Kopfschmerzen und Lichtempfindlichkeit. Die frühkindliche Entwicklung verlief unauffällig, Impfungen erfolgten entsprechend den STIKO-Empfehlungen. Zudem wurden im April und Mai 2007 FSME-Impfungen vorgenommen. Die Familienanamnese war bis auf eine multiple Sklerose großmütterlicherseits bezüglich Autoimmunerkrankungen, Immundefekten oder Krebserkrankungen leer.
Lichttherapie ausreichend. Erst bei Progredienz der Erkrankung bzw. tiefreichenden gelenkübergreifenden Verlaufsformen, ist eine Systemtherapie indiziert. Ermutigende Resultate konnten in verschiedenen Studien unter einer niedrig dosierten Methotrexat-Therapie ( $15 \mathrm{mg} /$ Woche) erreicht werden. Wir stellen eine 9-jährige Patientin mit zirkumskripter linearer Sklerodermie und drohender Kontraktur im Mittelgelenk des linken Ringfingers vor, bei der sich unter der systemischen Gabe von MTX $\left(10 \mathrm{mg} / \mathrm{m}^{2}\right)$ und Steroidpulstherapie ein deutlicher Rückgang der Krankheitsaktivität zeigte.

\section{Erstbefund}

Es zeigte sich in der linken Armbeuge eine ausgedehnte, ca. handtellergroße weißliche, indurierte atrophische Plaque umgeben von einem livid-rötlichen Randsaum. Einzelne kleinere Herde fanden sich auch entlang des Unterarms sowie am Handrücken.

Am Ringfinger der jungen Patientin sah man neben einer zirkulär angeordneten atrophischen Plaque eine deutliche Schwellung im Bereich des Mittelgelenks mit beginnender Beugekontraktur und eingeschränkter Beweglichkeit ( $\bullet$ Abb. 1). Sonstige Gelenke waren sämtlich aktiv und passiv frei beweglich. Die orientierende internistische und neurologische Untersuchung verlief altersentsprechend unauffällig.

\section{Befunde diagnostischer Untersuchungen $\nabla$}

Unauffällig bzw. altersentsprechend waren Differenzialblutbild, Leber-/Nierenparameter, Blutzucker, CRP, Urin- und Gerinnungsstatus, infektiologische Serologie und Autoantikörperdiagnostik. 


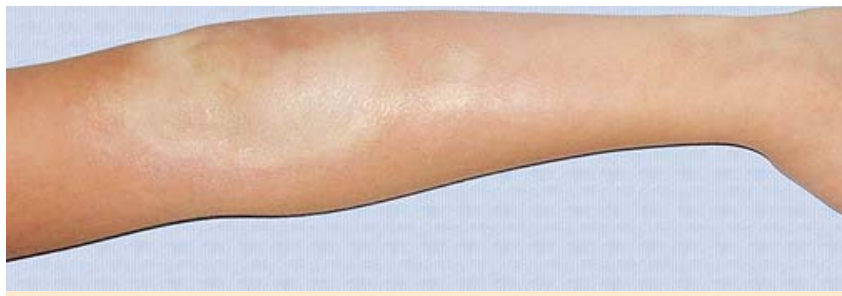

Abb. 1 Indurierte, atrophische Plaques mit livid-rötlichem Randsaum am linken Unterarm sowie initiale Beugekontraktur des linken Ringfingers bei linearer zirkumskripter Sklerodermie. Erstvorstellung der Patientin, vor Therapiebeginn.

\section{Ergänzende Untersuchungen \\ $\nabla$}

Sonografisch zeigte sich im Bereich der sichtbar veränderten Hautabschnitte eine nahezu homogene angehobene Echogenität von Haut und subkutanem Fettgewebe mit verwaschener HautSubkutisgrenze. Tiefenausdehnung im Mittel $5 \mathrm{~mm}$. Weiterführende röntgenologische und augenärztliche Untersuchungen waren unauffällig.

Bei typischem klinischem Befund wurde auf die Entnahme einer Probebiopsie verzichtet.

\section{Therapie und Verlauf \\ $\nabla$}

Anhand der Anamnese und des hier typischen klinischen Befundes diagnostizierten wir eine lineare zirkumskripte Sklerodermie.

Aufgrund des ausgedehnten progredienten Hautbefundes und der starken Aktivität der Erkrankung mit drohender Zunahme der Bewegungseinschränkung des linken Ringfingers entschlossen wir uns für die Einleitung einer Hochdosis-Steroid-Pulstherapie mit nachfolgender MTX-Erhaltungstherapie. Initial erhielt die junge Patientin in der ersten und zweiten Woche eine Methylprednisolon-Hochdosistherapie von $500 \mathrm{mg} / \mathrm{d}$ i.v. an drei aufeinanderfolgenden Tagen. Zwischen den beiden Zyklen und am Ende der Hochdosistherapie wurde Prednisolon gewichtsadaptiert $(0,5 \mathrm{mg} / \mathrm{kgKG})$ p. o. in absteigender Dosierung verabreicht.

Ab Tag 15 setzte die Erhaltungstherapie mit 12,5 mg MTX p.o. $\left(10 \mathrm{mg} / \mathrm{m}^{2}\right) 1 \times$ wöchentlich unter Folsäuresubstitution von $0,8 \mathrm{mg}$ am Folgetag ein, welche insgesamt 12 Monate andauerte. Eine engmaschige medizinische Betreuung und Kontrolle während der Therapie wurde durchgeführt.

Die Therapie wurde insgesamt gut toleriert, bis auf gelegentliche kurzzeitige Diarrhöen und Übelkeit traten keine interkurrenten Erkrankungen auf.

In der aktuellen Aufnahme von Dezember 2008 zeigt sich eine deutliche Aktivitätsabnahme der Erkrankung mit Rückgang der Rötung und Induration sowie eine resultierende postinflammatorische Hyperpigmentierung ( $\bullet$ Abb. 2 u. 3). Die Beweglichkeit des Ringerfingers zeigte sich in der klinischen Untersuchung deutlich gebessert.

In der sonografischen Kontrolle ließ sich eine deutliche Rückbildung der beschriebenen Veränderungen, insbesondere der Tiefenausdehnung, von zuvor $5 \mathrm{~mm}$ im Mittel auf $1 \mathrm{~mm}$ nachweisen.

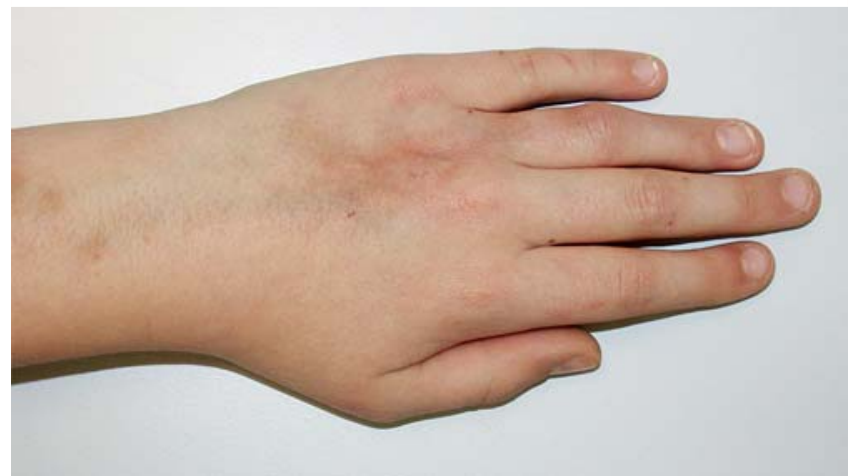

Abb. 2 Rückläufiger Lokalbefund mit Wiederherstellung der Beweglichkeit des linken Ringfingers nach Hochdosis-Steroid-Pulstherapie und nachfolgender MTX-Erhaltungstherapie.

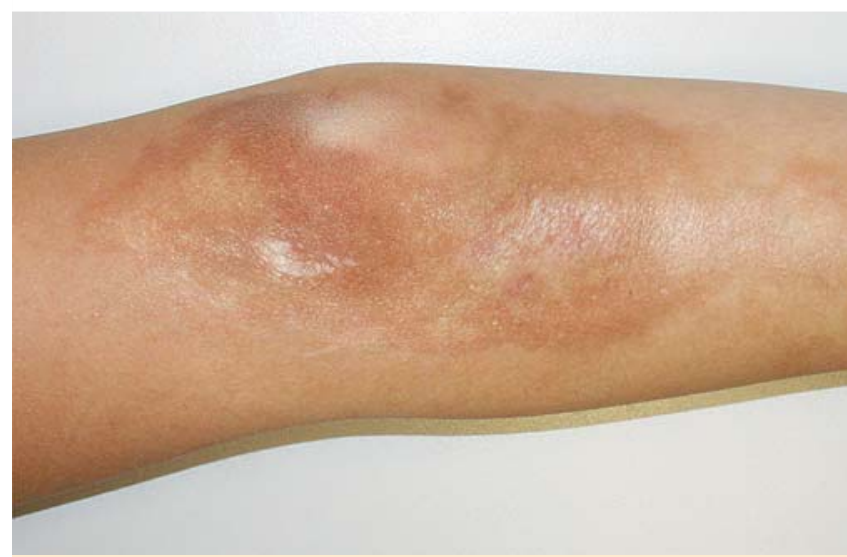

Abb. 3 Aktivitätsabnahme der Erkrankung mit resultierender postinflammatorischer Hyperpigmentierung nach Hochdosis-Steroid-Pulstherapie und nachfolgender MTX-Erhaltungstherapie.

\section{Besprechung}

$\nabla$

Die zirkumskripte Sklerodermie ist eine seltene entzündliche sklerosierende Erkrankung. Die Inzidenz wird in einigen Studien auf 0,4 bis 1 pro 100000 Einwohner geschätzt. Die hier beschriebene lineare Verlaufsform ist nach dem Plaque-Typ (36\%) mit ca. 25-30\% der zweithäufigste Subtyp der zirkumskripten Sklerodermie. Neueste Untersuchungen an einem Patientenkollektiv von 245 Patienten zeigten, dass Kinder und Erwachsene weitgehend gleichmäßig betroffen sind. In früheren kleineren Kollektiven wurde das Verhältnis von 2:1 Kindern zu Erwachsenen beobachtet. Neben der Haut können bei der zirkumskripten Sklerodermie auch extrakutane Beteiligungen auftreten. Dabei sind neben muskuloskelettalen (z.B. Arthralgien) auch gastrointestinale, neurologische, pulmonale und vaskuläre Manifestationen beschrieben worden [15]. Bei der linearen Verlaufsform kann es in dem betroffenen Körperteil zu Wachstumsstörungen, Fehlstellungen und Verkürzungen von Knochen und Muskulatur kommen. Insgesamt kann dies häufig zu erheblichen körperlichen und auch psychischen Konsequenzen führen. Auch neurologische Symptome (z.B. Kopfschmerzen, periphere Neuropathien) sowie ophthalmologische Erkrankungen (z.B. Episkleritis, Glaukom, Uveitis) werden bei der linearen Verlaufsform vermehrt beobachtet [1]. In der kürzlich veröffentlichten Studie von Leitenberger et al. [14] zeigten sich daneben auch Assoziationen mit Systemerkrankungen, nicht selten mit Autoimmunkrankheiten und rheumatologischen Erkrankungen. Dabei wiesen Kinder im Vergleich 
zu den erwachsenen Probanden deutlich weniger (4,4\% vs. 30,1\%) begleitende Autoimmunerkrankungen auf. Ob Kinder mit Morphea später im Erwachsenenalter eine erhöhte Rate an Autoimmunkrankheiten aufweisen, bleibt noch zu untersuchen.

Die Ätiologie der zirkumskripten Sklerodermie ist noch weitestgehend unbekannt. Diskutiert werden genetische (Korrelation mit HLA-B8, DR1, DR5), immunologische, hormonelle, virale, toxische, traumatische, medikamentöse, neurogene oder vaskuläre Faktoren. Jüngere Studienergebnisse zeigen eine hohe Korrelation mit einer vorangegangenen Borrelieninfektion $[2,16]$. Die Diagnose der linearen Sklerodermie stützt sich vor allem auf das charakteristische, klinische Bild. Die histologische Untersuchung unterstützt die klinische Diagnose. Im Gegensatz zur systemischen Sklerodermie sind die antinukleären Antikörper meist unauffällig $[3,14]$. Aufgrund der spontanen Abheilungstendenz ist zunächst therapeutische Zurückhaltung geboten. Meist ist eine Lokalbehandlung mit Glukosteroiden, Vitamin-D3-Analoga, UVA1- oder PUVA-Lichttherapie ausreichend [4-7]. Erst bei Progredienz der Erkrankung ist eine Systemtherapie indiziert. Klinisch unterschiedliche Ergebnisse wurden mit Penicillamin, Antimalariamitteln, Retinoiden, Calcipotriol, Cyclosporin und Interferon gamma beschrieben [8], welche vor allem bei tiefreichenden Verlaufsformen nur begrenzte Erfolge zeigten [9].

Ermutigende Resultate konnten jedoch in verschiedenen Studien unter einer niedrig-dosierten Methotrexat-Therapie (15 mg/Woche) erreicht werden $[8,10,11]$. Trotz des jungen Alters der Patientin entschlossen wir uns aufgrund der Befundprogredienz und der drohenden Kontraktur des linken Ringfingers im Mittelgelenk zur Einleitung einer Hochdosis-Steroid-Pulstherapie mit nachfolgender MTX-Erhaltungstherapie, angelehnt an die Studienergebnisse von Weibel et al. [12]. Anhand einer retrospektiven Untersuchung an 34 Kindern unter Kombinationstherapie von MTX und Steroiden zeigte sich bereits zu Therapiebeginn bei 94\% der Patienten ein Sistieren der Erkrankung. 71\% der behandelten Kinder wiesen auch zum Zeitpunkt der letzten Kontrolle, nach 2 bis 7 Jahren, keinerlei Krankheitsaktivität mehr auf.

Auch bei unserer jungen Patientin zeigte sich unter der eingeleiteten Therapie eine deutliche Aktivitätsabnahme der Erkrankung bei insgesamt nur geringen Nebenwirkungen (Grad I und II nach WHO).

Der genaue Wirkmechanismus von niedrig dosiertem Methotrexat auf die Fibrose ist jedoch bis heute unklar. Vermutet wird ein direktes Einwirken auf die Fibroblasten der Haut oder ein generell antiinflammatorischer Effekt [13].

Im Hinblick auf die beschriebene Datenlage sollte auch bei Kindern und Jugendlichen bei Vorliegen einer zirkumskripten linearen oder profunden Sklerodermie und damit verbundenen drohenden funktionellen Beeinträchtigungen frühzeitig eine Systemtherapie mit einer initialen Hochdosis-Steroid-Pulstherapie und Erhaltungstherapie mit MTX in Erwägung gezogen werden. Aufgrund möglicher Nebenwirkungen sollte diese Behandlung unter engmaschigen klinischen und labormedizinischen Kontrollen erfolgen.

\section{Abstract}

\section{Linear Scleroderma}

$\nabla$

The localized scleroderma is a continuous form of Morphea characterized with a localized unilateral arrangement on arms or legs. It often affects underlying muscles and bones and can cause significant atrophy, growth retardation, irreversible structural deformities, joint contractures and several functional, cosmetic and psychological disabilities. Until now the etiology is unknown/unexplained. Mostly a local therapy with corticosteroids, vitamin-D3-drugs or a phototherapy with UVA or PUVA is enough to stop the progress of the disease. A systemic therapy is only indicated in case of progress with consequent deep-tissue defects. Encouraging results could be attained with a low-dose MTX-therapy.

We would like to present a 9-year-old girl with localized scleroderma and imminent loss of function of the middle-finger-articulation. After receiving a low-dose-MTX this defect clearly declined.

\section{Literatur}

1 Zulian F. New developments in localized scleroderma. Curr Opin Rheumatol 2008; 20: 601-607

2 Hercogova J. Borrelia burgdorferi: a protagonist in Lyme disease, a bystander in morphea? J Eur Acad Dermatol Venereol 2002; 16: 98 - 99

3 Fabri M, Hunzelmann N. Differenzialdiagnose der Sklerodermien und Pseudosklerodermien. JDDG 2007; 11: 977-984

4 Kerscher M, Volkenandt M, Meurer M, Lehmann P, Plewig G, Röcken M. Treatment of localised scleroderma with PUVA bath photochemotherapy. Lancet 1994; 343: 1233

5 Kerscher M, Dirschka T, Volkenandt M. Treatment of localised scleroderma by UVA1 phototherapy. Lancet 1995; 346: 1166

6 Kerscher M, Volkenandt M, Gruss C, Reuther T, Kobyletzki G von, Freitag $M$, Dirschka T, Altmeyer P. Low-dose UVA1 phototherapy for treatment of localized scleroderma. J Am Acad Dermatol 1998; 38: 21 - 26

7 Kreuter A, Gambichler T, Avermaete A, Jansen T, Hoffmann M, Hoffmann $K$, Altmeyer P, Kobyletzki G von, Bacharach-Buhles M. Combined treatment with calcipotriol ointment and low-dose ultraviolet A1 phototherapy in childhood morphea. Pedtr Dermatol 2001; 18: 241 - 245

8 Kreuter A, Gambichler T, Breuckmann F, Rotterdam S, Freitag M, Stuecker $M$, Hoffmann K, Altmeyer P. Pulsed high-dose corticosteroids combined with low-dose methotrexate in severe localized scleroderma. Arch Dermatol 2005; 141: 847-852

9 Hunzelmann N, Anders S, Fierlbeck G, Hein R, Herrmann K, Albrecht M, Bell S, Muche R, Wehner-Caroli J, Gaus W, Krieg T. Double-blind, placebo-controlled study of intralesional interferon gamma for the treatment of localized scleroderma. J Am Acad Dermatol 1997; 35: 433 435

10 Joly P, Bamberger N, Crickx B, Belaich S. Treatment of severe forms of localized scleroderma with oral corticosteroids: Follow-up study on 17 patients. Arch Dermatol 1994; 130: 664-665

11 Seyger MMB, van der Hoogen FHJ, de Boo T, de Jong EMGJ. Low-dose methotrexate in the treatment of the widespread morphea. J Am Acad Dermatol 1998; 30: 220-225

12 Weibel L, Sampaio MC, Visentin MT, Howell KJ, Woo P, Harper JI. Evaluation of methotrexate and corticosteroids for the treatment of localized scleroderma (morphoea) in children. Br J Dermatol 2006; 155: 1013 1020

13 van den Hoogen FH, van den Kraan PM, Boerbooms AM, van den Berg WB, van Lier HJ, van de Putte LB. Effects of methotrexate on glycosaminoglycan production by scleroderma fibroblasts in culture. Ann Rheum Dis 1993; 52: 758-776

14 Leitenberger JJ, Cayce RL, Haley RW, Adams-Huet B, Bergstresser PR, Jacobe HT. Distinct Autoimmune Syndromes in Morphea. Arch of Dermatology 2009; 5: 545-550

15 Christen-Zaech S, Hakim MD, Afsar FS, Paller AS. Pediatric morphea (localized scleroderma): Review of 136 patients. J Am Acad Dermatol 2008; 9: 385-396

16 Prinz JC, Kutasi Z, Weisenseel P, Poto L, Battyani Z, Ruzicka T. „Borreliaassociated early-onset morphea“: a particular type of scleroderma in childhood and adolescence with high titer antinuclear antibodies? Results of a cohort analysis and presentation of three cases. J Am Acad Dermatol 2009; 2: 248-255 